Open Access

\title{
Senescent mesenchymal stem cells promote colorectal cancer cells growth via galectin-3 expression
}

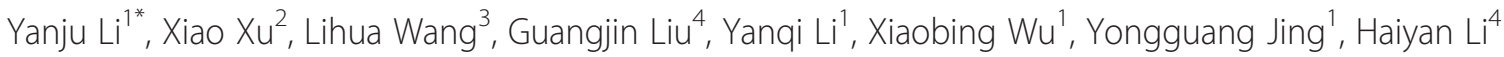 \\ and Guihua Wang ${ }^{1}$
}

\begin{abstract}
Background: Cellular senescence is linked to aging and tumorigenesis. The senescence of mesenchymal stem cells (MSCs) may influence the tumor growth, metastasis, and angiogenesis by secreting a variety of cytokines and growth factors.

Results: The conditioned media of adipose derived MSCS (AD-MSCs) stimulated the proliferation of human LoVo colorectal-cancer cells, and the replicative senescent MSCs had the more obvious effects in comparison to that of premature AD-MSCs. Analysis of the factors secreted in the MSCs culture media determined that senescent MSCs expressed and secreted high levels of galectin-3. Galectin-3 expression correlated with the stimulatory effect of senescent AD-MSCs on LoVo cells proliferation, as knockdown of galectin-3 in senescent AD-MSCs significantly reversed the effect of MSCs-mediated growth stimulation of LoVo cells. Furthermore, the simultaneous addition of recombinant galectin-3 to the co-culture systems partially restored the tumor-promoting effect of the senescent AD-MSCs. Analysis of the mechanisms of senescent MSCs and galectin-3 on LoVo cells signal transduction determined that senescent MSCs and exogenous galectin-3 promoted cell growth by activating the mitogen-activated protein kinase (MAPK) (extracellular signal-regulated kinase [ERK]1/2) pathway.

Conclusions: Senescent MSCs may alter the tissue microenvironment and affect nearby malignant cells via cytokine secretion, and galectin-3 is an important mediator of senescent AD-MSC-mediated stimulation of colon cancer cell growth. Therefore, thorough assessment of AD-MSCs prior to their implementation in clinical practice is warranted.
\end{abstract}

Keywords: Cellular senescence, Mesenchymal stem cells, Galectin-3, LoVo cells

\section{Background}

Cellular senescence, a state of irreversible growth arrest, can be triggered by many mechanisms, including telomere shortening, epigenetic derepression of the cyclindependent kinase inhibitor 2A/ADP ribosylation factor (INK4a/ARF) locus, and DNA damage [1]. Cellular senescence is linked to aging and tumorigenesis, and has been proposed as a suppressive mechanism against the development of cancer [2, 3]. However, recent studies have revealed that cellular senescence also has tumorpromoting effects, and the accumulation of senescent

\footnotetext{
* Correspondence: lyj8181@163.com

'Department of Hematology, Affiliated Hospital of Guiyang Medical College, No. 28, Guiyi Street, Yunyan District, Guiyang, Guizhou Province 550004, China

Full list of author information is available at the end of the article
}

cells during the aging process in vivo may contribute to the age-related increase in cancer incidence [4].

Mesenchymal stem cells (MSC), which possess broad and potent multilineage differentiation potential and potent immunoregulatory properties, are the optimal source for stem cell transplant in clinical applications $[5,6]$. Similar to other adult somatic cells, long-term culture in vitro leads to MSC senescence, which results in growth arrest and reduced differentiation [7, 8]. The replicative senescence of MSCs is evinced by telomere shortening, enlargement and flattening of the characteristic stem cell morphology, reduced proliferation rate, and altered secretory profile $[8,9]$. The function of adult tissue-specific stem cells declines with age, and aged MSCs could be more deleterious as they can greatly

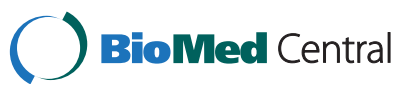

(c) 2015 Li et al. Open Access This article is distributed under the terms of the Creative Commons Attribution 4.0 International License (http://creativecommons.org/licenses/by/4.0/), which permits unrestricted use, distribution, and reproduction in any medium, provided you give appropriate credit to the original author(s) and the source, provide a link to the Creative Commons license, and indicate if changes were made. The Creative Commons Public Domain Dedication waiver (http://creativecommons.org/publicdomain/zero/1.0/) applies to the data made available in this article, unless otherwise stated. 
impair tissue homeostasis and repair $[9,10]$. MSCs from aged patients with coronary artery disease have impaired angiogenic potential and reduced proangiogenic factor secretion [11]. Senescent MSCs may contribute to the physiological decline in tissue homeostasis and to the increased risk of neoplasm during aging [9].

Recently report showed that MSCs stimulated invasion, survival and tumorigenesis of colorectal cancer cells through the release of soluble NRG1, activating the HER2/HER3-dependent PI3K/AKT signalling cascade in colorectal cancer cells [12]. In this study, we showed that replicative senescent AD-MSCs significantly promoted the proliferation of LoVo colorectal-cancer cells in comparison to premature AD-MSCs, and the expression of galectin-3, a powerful modulator of cell migration and spread in carcinoma cells, correlated with the stimulatory effect of senescent AD-MSCs on colorectal cancer cells proliferation. Therefore, thorough assessment of AD-MSCs prior to their implementation in clinical practice is warranted.

\section{Results}

\section{Characterization of P30-MSCs}

In the first few generations, the AD-MSCs exhibited fibroblast-like morphology. After 30 passages, the MSCs appeared longer and larger, with accumulation of granular cytoplasmic inclusions. The percentage of positive SA- $\beta$-Gal staining was increased significantly in the replicative P30-MSCs (Fig. 1a). The AD-MSCs were also capable of osteogenic and adipogenic differentiation when cultured in the appropriate inducing media. Assessment of the degree of osteogenic and adipogenic differentiation via Alizarin Red S and Oil Red O staining, respectively, revealed a sharp decline in the adipogenic and osteogenic potential of P30-MSCs compared to P3MSCs (Fig. 1b). CCK-8 analysis showed that the proliferation potential of AD-MSCs declined significantly with cell replicative passaging (Fig. 1c). Western blot analysis of $\mathrm{p} 53$ and $\mathrm{p} 21$ expression in the AD-MSCs showed that p53 and p21 expression increased gradually with passage (Fig. 1d). Flow cytometric analysis demonstrated that both P3-MSCs and P30-MSCs were positive for CD73, CD90, and CD105, and negative for CD34, CD45, and HLA-DR (data not shown).

\section{CM-P30 promoted colon cancer cell proliferation}

To determine the effect of P30-MSCs on colon cancer cell growth, LoVo cells were cultured with concentrated CM-P3 or CM-P30 for $48 \mathrm{~h}$. As shown in Fig. 2a-c, the conditioned medium of MSCs can promoted LoVo cell proliferation, and the LoVo cells cultured with CM-P30 spread and grew faster than cells cultured with culture medium alone or with CM-P3. However if the cells were incubated for $48 \mathrm{~h}$, both of CM-P3 and CM-P30 treated colon cancer cell groups achieved similar proliferation levels.

\section{Galectin-3 contributed to P30-MSC stimulation of colon cancer cell growth}

Accumulated evidence indicates that galectin-3 is closely involved in tumor cell proliferation, transformation, migration, invasion, and metastasis [13]. We analyzed galectin-3 expression in CM-P3 or CM-P30 with quantitative PCR (Q-PCR) and ELISA, and found that both mRNA and protein levels of galectin-3 were significantly upregulated in the AD-MSCs during senescence (Fig. 3a-b), suggesting that galectin-3 may have been involved in the P30-MSCmediated growth stimulation of LoVo cells.

To examine whether galectin-3 secretion in P30-MSCs stimulates LoVo cell proliferation, we blocked galectin-3 expression in P30-MSCs with a galectin-3-specific siRNA. Q-PCR and ELISA data showed that LGALS3 mRNA expression was decreased and that galectin-3 secretion in CM-P30 was significantly reduced following siRNA treatment (Fig. 3c, d).

LoVo cells were incubated with 50 or $100 \mathrm{ng} / \mathrm{ml} \mathrm{recom-}$ binant galectin-3 for 24 hours, and the proliferation of LoVo cells were evaluated by CCK-8. As Fig. 4a showed that the rgalectin-3 enhanced the growth of LoVo cells. LoVo cells were then incubated with CM-P30 pre-treated with the galectin-3 siRNA or NC, and the knockdown of galectin-3 in senescent AD-MSCs significantly reversed the effect of MSCs-mediated growth stimulation of LoVo cells (Fig. 4b). Furthermore, the simultaneous addition of $100 \mathrm{ng} / \mathrm{ml}$ recombinant galectin-3 to the co-culture systems partially restored the tumor-promoting effect of the senescent AD-MSCs.

\section{P30-MSCs promoted ERK1/2 activation in colon cancer cells}

As reported previously [14], exogenous galectin-3 induces the extracellular signal-regulated kinases (ERK1/2) phosphorylation in cancer cells, and the activation of ERK $1 / 2$ are associated with cancer cell proliferation and survival $[15,16]$. Our western blot data were showed in Fig. 5a, the CM of MSCs promoted ERK1/2 phosphorylation in the LoVo cells and that CM-P30 had a greater stimulative effect on ERK1/2 activation. Moreover, the phosphorylation of ERK1/2 induced by CM-P30 of MSCs were aborted by U0126, the specific inhibitor of MEK1/2, suggesting that the signal was transferred through a specific Raf-MEK1/2ERK1/2 pathway to activate ERK1/2. We then knocked down galectin-3 expression in the P30-MSCs and compared the promoter effect of the CM-P30 on ERK1/2 phosphorylation to that of the CM of MSCs treated with $\mathrm{MSC}_{\mathrm{NC}}$. Galectin-3 knockdown diminished the CM-P30induced ERK1/2 phosphorylation; however, the addition of 


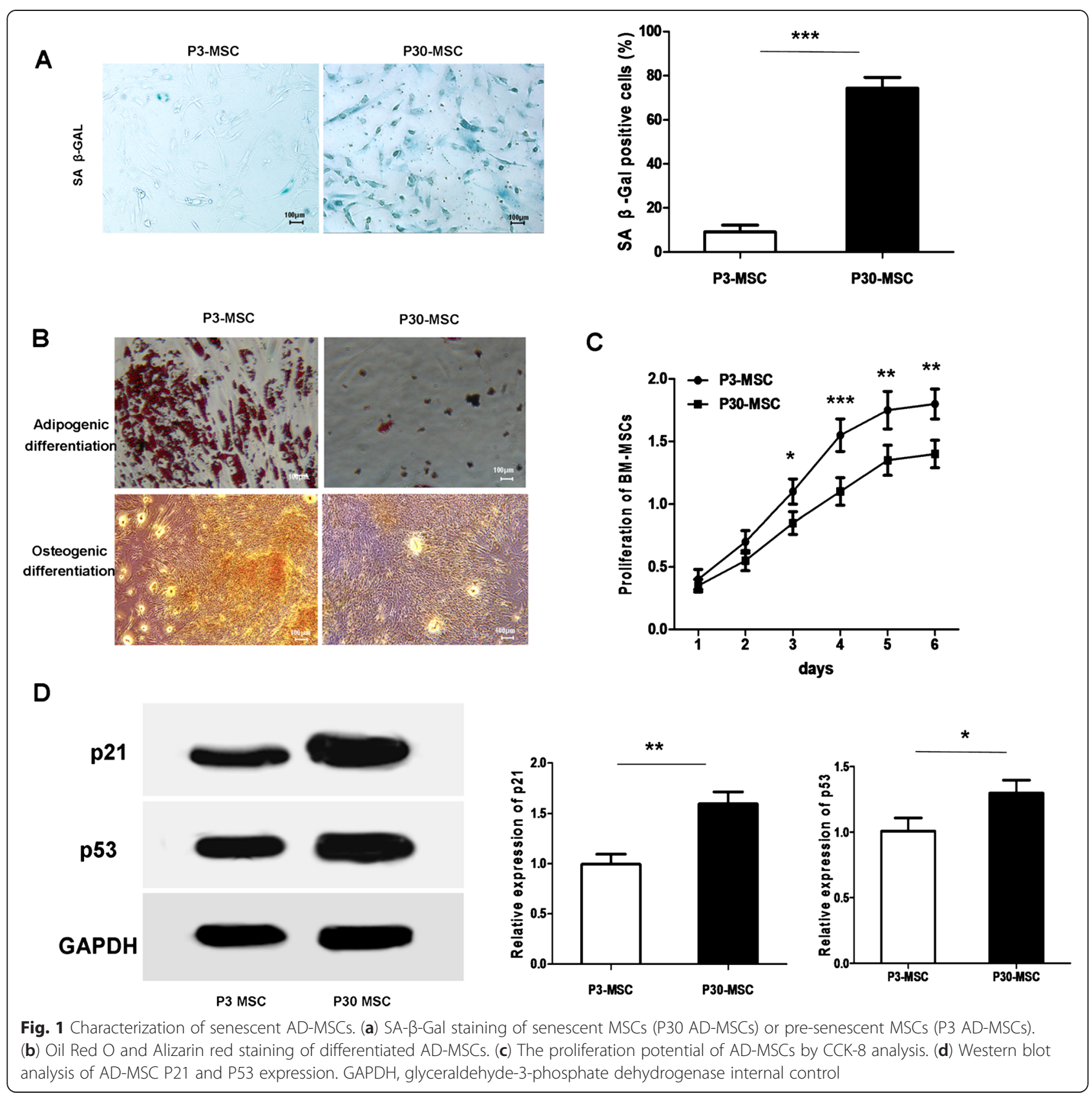

exogenous galectin-3 to the CM restored ERK1/2 activation in the LoVo cells (Fig. 5b).

\section{Discussion}

Recent studies have shown that a pool of molecules secreted by senescent cells, referred to as having the senescence-associated secretory phenotype (SASP), is associated with arrest of cell proliferation and may contribute to it via the autocrine/paracrine pathways $[10,17]$. Our data revealed that the MSCs had the typical senescence-associated characteristics and SASP after repeated passage, marked by the appearance of senescence- associated morphological features, decreased proliferation, SA- $\beta$-Gal positivity, induced p53 and p21 expression, and increased galectin-3 expression. We then showed that CM-P30 promoted colon cancer cell proliferation. In the co-culture experiments, we demonstrated that galectin-3 mediated the promoter effects of AD-MSCs on colon cancer cell proliferation to some extent, as specific knockdown of galectin-3 with siRNA significantly reversed the MSC-mediated stimulation of colon cancer cell growth.

The tumor microenvironment is increasingly regarded as an important regulator of malignant progression of cancer cells [18]. MSCs may secrete a variety of cytokines and 


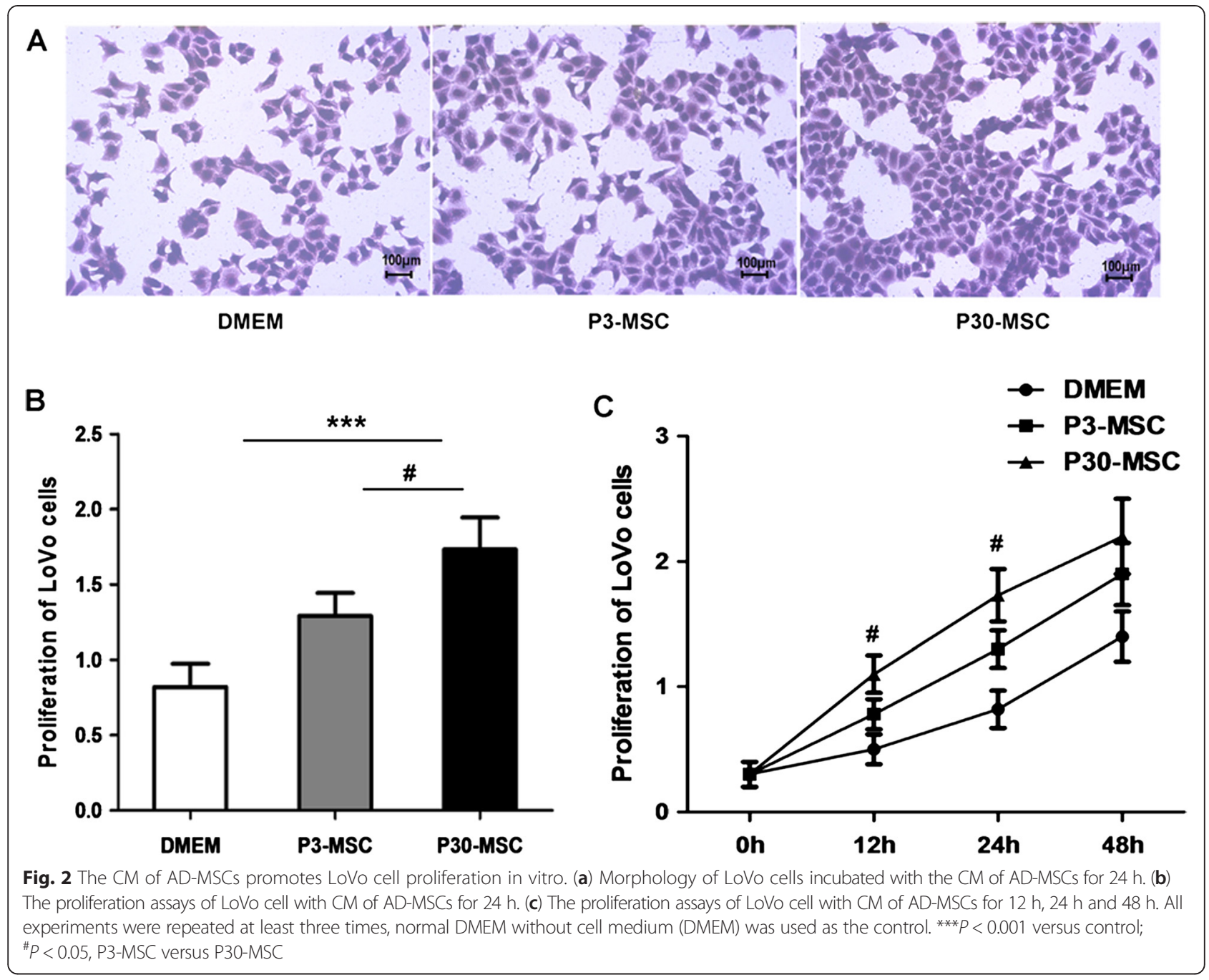

growth factors that influence tumor growth, metastasis, and angiogenesis [19, 20]. Karnoub et al. [19] showed that MSCs play a pivotal role in colon cancer progression and metastasis; when recruited into breast cancer stroma, bone marrow MSCs tended to facilitate breast cancer cell metastasis and regulate cancer stem cell behavior via the secretion of the chemokine CCL5. Recent studies have shown that the CM of MSCs enhances tumor growth, indicating that the factors secreted by MSCs have profound effects on "reprogramming" tumor growth [18, 21], and the senescent umbilical cord MSCs promoted the proliferation and migration of breast cancer cells [22].

Of the MSC SASP factors, we found that galectin-3 was an important mediator of cancer-promoting activity. A unique chimera-type member of the $\beta$-galactoside-binding soluble lectin family with a molecular mass of $29-35 \mathrm{kD}$, galectin-3 is implicated in a variety of biological functions $[23,24]$. Previously, Liu et al. showed that galectin-3 is secreted into the CM of UC-MSCs and is involved in the immunosuppressive function of UC-MSCs [25]. In addition, galectin-3 expression in most cancer cells is increased, and is associated with growth and metastases in pancreatic and breast cancer systems [26]. A recent report [27] showed that galectin-3 is involved in the nicotineinduced promotion of apoptosis resistance of breast cancer cells and that it promotes cancer cell growth and protects cells from apoptosis induced by chemotherapeutic drugs. Baptiste et al. [28] showed that galectin-3 was a powerful modulator of cell adhesion and spread in breast carcinoma cells and that the exogenous addition of recombinant galectin-3 promoted the growth of galectin-3-null cells. However, our data showed that recombinant galectin-3 has a weaker effect on cancer cell growth than senescent MSCs, suggesting that other cytokines secreted from senescent MSCs may be associated with the stimulation of colon cancer cell growth.

Lastly, we analyzed the mechanisms of senescent MSCs and galectin- 3 on colon cancer cell signal transduction, 


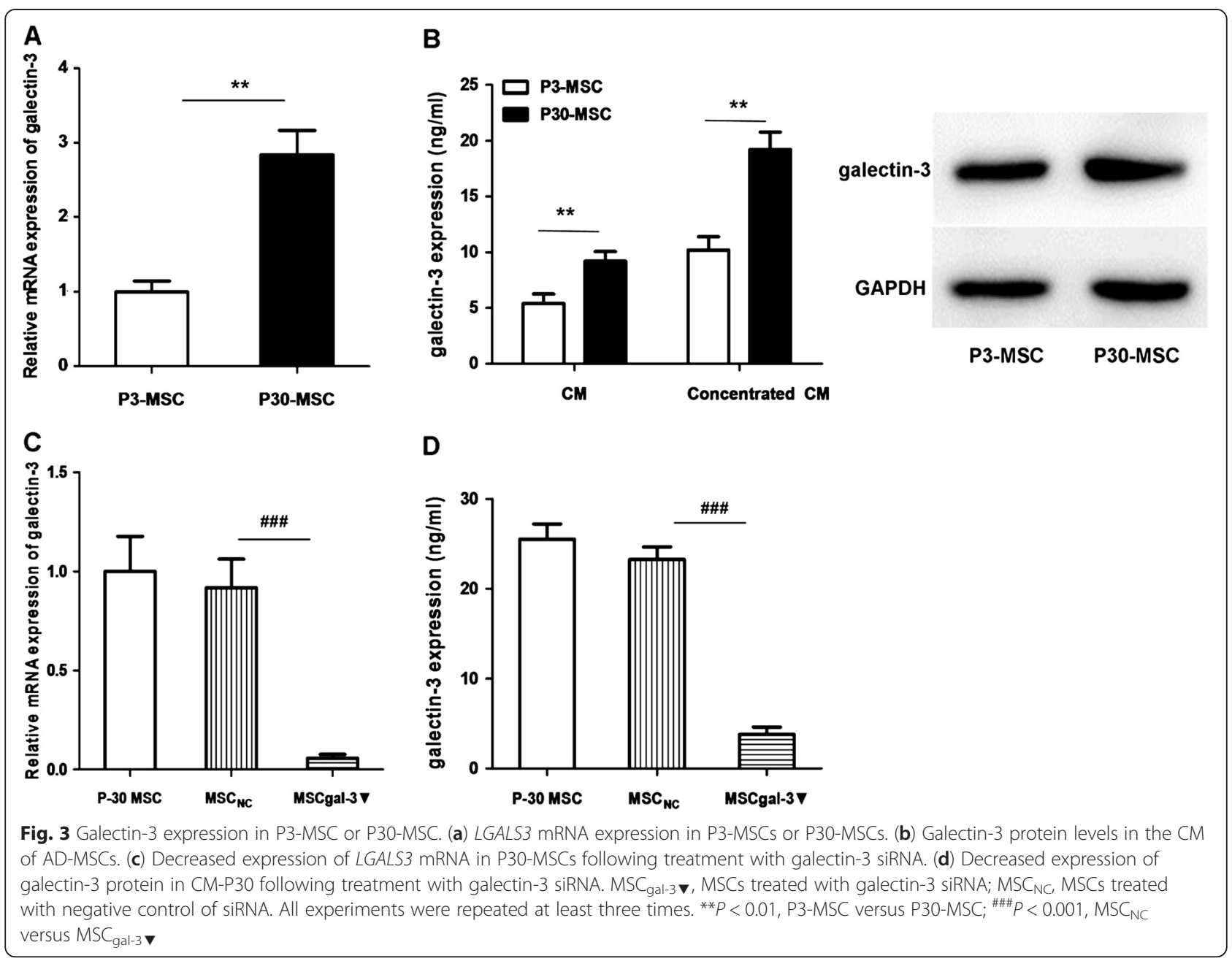

finding that senescent MSCs and exogenous galectin-3 promoted colon cancer cell growth by activating the MAPK (ERK1/2) pathway. The extracellular signalregulated kinases (ERK1/2) which are serine/threonine protein kinases, as one of the most important regulator of key cellular processes [15], involved in carcinogenesis due to their ability to stimulate cell proliferation and survival [16]. The MAPK pathways are activated by diverse extracellular and intracellular stimuli including peptide growth factors, cytokines, hormones, and various cellular stressors [29]. Gao et al. [14] showed that exogenous galectin-3 induces ERK1/2 phosphorylation in cancer cells, and our results are consistent with this reports.

In summary, our study suggests that senescent MSCs may alter the tissue microenvironment and affect nearby malignant cells via cytokine secretion, and that galectin-3 is an important mediator of senescent AD-MSC-mediated stimulation of colon cancer cell growth. Therefore, thorough assessment of AD-MSCs prior to their implementation in clinical practice is warranted.

\section{Materials and methods}

Isolation and preparation of senescent AD-MSCs

The AD-MSCs were isolated and obtained by density gradient centrifugation, approved by the Ethics Committee of the Affiliated Hospital of Guiyang Medical College. Briefly, AD-MSCs were isolated and cultured according to a collagenase digestion protocol. Mononuclear cells were plated in $\alpha$-minimum essential medium (Invitrogen, Carlsbad, CA, USA) containing $10 \%$ fetal bovine serum (FBS) (HyClone, Utah, USA), and cultured in a humidified incubator containing $5 \% \mathrm{CO} 2$ at $37{ }^{\circ} \mathrm{C}$.

AD-MSCs were further cultivated to confluence and were amplified, and replicative senescent cells were passaged serially over 30 passages when the typical senescenceassociated morphological features appeared. Passage 3 (P3) and P30 cells were referred to as "young" (P3-MSCs) and "senescent” MSCs (P30-MSCs), respectively.

\section{Characterization of senescent AD-MSCs}

Senescence-associated $\beta$-galactosidase (SA- $\beta$-Gal) staining was performed using an SA- $\beta$-Gal staining kit (Cell 

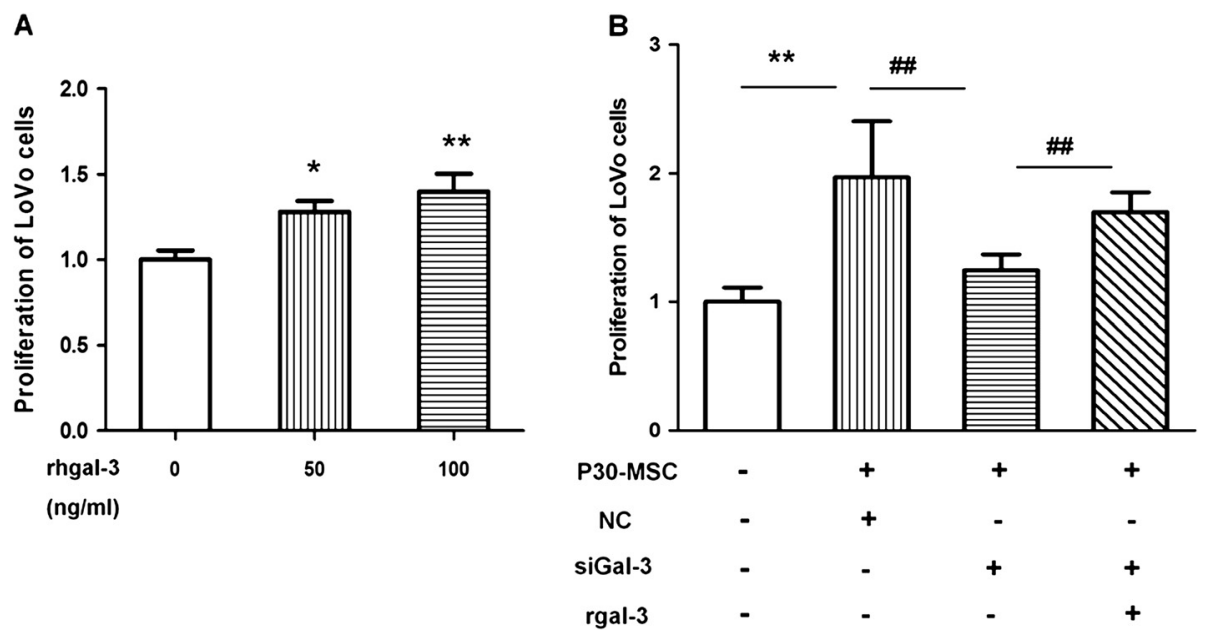

Fig. 4 Galectin-3 is an important mediator of P30-MSC-mediated stimulation of LoVo cell growth. (a) Proliferation of LoVo cells incubated with 50 or $100 \mathrm{ng} / \mathrm{ml}$ recombinant galectin-3. (b) Proliferation of LoVo cells incubated with CM of P30-MSCs, which treated with galectin-3 siRNA or NC. Control, normal DMEM without cell medium; siGal-3, galectin-3 siRNA; rgalectin-3, recombinant galectin-3. All experiments were repeated at least three times. ${ }^{*} P<0.05,{ }^{* *} P<0.01$ versus control; ${ }^{\# \#} P<0.01$, versus siGAL-3

Signaling Technology, Beverly, MA, USA) according to the manufacturer's instructions. For the proliferation analysis, MSCs were counted every 24 hours for 5 days using a cell counting kit-8 (CCK-8) (DoJinDo, ShangHai, China). All experiments were performed three times.

AD-MSCs surface marker analysis by flow cytometry was performed on a FACSCalibur unit (Becton Dickinson
Biosciences, San Jose, CA, USA); cells were stained with phycoerythrin-conjugated antibodies against CD105, CD73, CD90, CD45 and HLA-DR (Becton Dickinson Biosciences). For osteogenic and adipogenic differentiation, MSCs were incubated with MesenCult Osteogenic or Adipogenic Stimulatory Medium (STEMCELL Technologies, Vancouver, Canada) for 2-3 weeks. Osteogenic and adipogenic
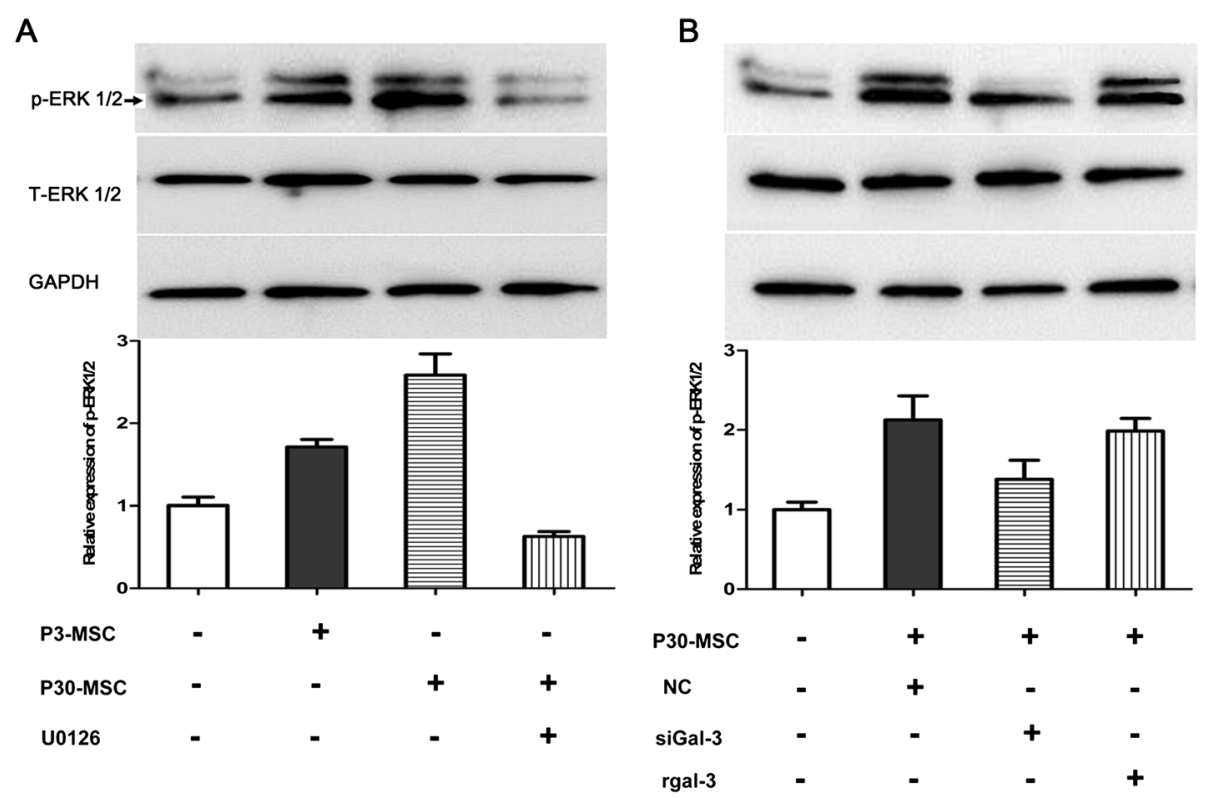

Fig. 5 Western blot analysis of P30-MSC and exogenous galectin-3 promotion of ERK1/2 activation in LoVo cells. (a) the CM of MSCs promoted ERK1/2 phosphorylation in the LoVo cells, which were aborted by U0126 for 60 min treatment. (b) LoVo cells incubated with CM of P30-MSCs, which treated with or without galectin-3 siRNA.siGal-3, galectin-3 siRNA; rgalectin-3, recombinant galectin-3; GAPDH was used as the internal control. All experiments were repeated at least three times. ${ }^{\#} P<0.01$ versus control; ${ }^{* *} P<0.01$ versus siGAL-3 
differentiation was evaluated using Alizarin Red S and Oil Red O (Sigma-Aldrich, St. Louis, MO, USA) staining, respectively.

p21 and p53 protein levels were analyzed by western blotting. Proteins were extracted from $1 \times 10^{6} \mathrm{P} 3$-MSCs or P30-MSCs with $1 \mathrm{ml}$ radioimmunoprecipitation assay (RIPA) buffer (Sigma-Aldrich), separated by $10 \%$ sodium dodecyl sulfate-polyacrylamide gel electrophoresis (SDSPAGE), and transferred to Immobilon polyvinyl difluoride (PVDF) membranes. The membranes were incubated with antibody against p21 and p53 (Abcam, Cambridge, MA, USA), followed by goat anti-rabbit horseradish peroxidase (HRP)-conjugated antibody (Santa Cruz Biotechnology, Santa Cruz, CA, USA), and visualized with SuperSignal West Pico Chemiluminescent Substrate (Millipore, Billerica, MA, USA).

\section{Preparation of conditioned medium}

To harvest the MSC conditioned medium (CM), $80 \%$ confluent P3-MSC and P30-MSC cultures were extensively washed with phosphate-buffered saline (PBS) and incubated in serum-free culture medium for $24 \mathrm{~h}$. Then, the CM from the P3-MSCs (CM-P3) and P30-MSCs (CM-P30) was collected and concentrated to $1 / 2 \times$ volume with an ultra-filtration membrane with a molecular weight cut-off of $3 \mathrm{kD}$ by centrifuging at $5000 \mathrm{rpm}$ for $30 \mathrm{~min}$ at $4{ }^{\circ} \mathrm{C}$. The concentrated $\mathrm{CM}$ was then filtered through a $0.22-\mu \mathrm{m}$ membrane and stored at $-80{ }^{\circ} \mathrm{C}$ until used.

\section{Cell proliferation assay of colon cancer cells}

The human LoVo colon cancer cell line was obtained from the American Type Culture Collection (ATCC). LoVo cells were established from a metastatic nodule resected from a 56-year-old Caucasian male colorectal adenocarcinoma patient [30].

The LoVo cells were cultured in phenol red-free Dulbecco's modified Eagle's medium (DMEM) (Invitrogen, Carlsbad, CA, USA) supplemented with $10 \%$ FBS; $2.5 \times$ $10^{3}$ cells/well were plated in $200 \mu$ l growth medium in $96-$ well plates. After $24 \mathrm{~h}$, the medium was replaced with concentrated conditioned medium of CM-P3 and CM-P30 as for $12 \mathrm{~h}, 24 \mathrm{~h}$ or $48 \mathrm{~h}$. Tumor cell proliferation was evaluated using Cell Counting Kit-8 according to the manufacturer's instructions.

\section{Analysis of galectin-3 expression in MSCs}

We analyzed galectin-3 (LGALS3) mRNA expression with real-time PCR: total RNA was isolated from $1 \times 10^{6}$ P3MSCs or P30-MSCs using TRIzol (Invitrogen) according to the manufacturer's instructions. First-strand complementary DNA was reverse-transcribed using a Reverse Transcriptase MIX Kit (Toyobo, Osaka, Japan). Quantitative real-time PCR was performed in an ABI 7500 Fast Real-Time PCR System (Applied Biosystems, Foster City,
CA, USA) using Fast SYBR Green PCR Master Mix (Applied Biosystems). We used the following primer pairs as reported previously [31]: $\beta$-actin (forward: 5 '-GAAGGTG AAGGTCGGAGTCA-3', reverse: 5'-GAAGATGGTGA TGGGATTTC-3') and LGALS3 (forward: 5' -CCAAAGA GGGAATGATGTTGCC-3', reverse: 5'-TGATTGTACT GCAACAAGTGAGC-3').

Galectin-3 levels in CM-P3 or CM-P30 were measured as described previously [25]. Briefly, the concentrated CM was analyzed using a Human Galectin-3 Platinum ELISA (enzyme-linked immunosorbent assay) Kit (eBioscience, San Diego, CA, USA) according to the manufacturer's instructions.

\section{Galectin-3 knockdown}

To knock down galectin-3 expression, P30-MSCs were transfected with predesigned small interfering RNA (siRNA) (Sigma-Aldrich) using Lipofectamine RNAiMAX reagent (Invitrogen). The respective siRNA sequences of MSCs are as follows: galectin-3 siRNA target site $\left(\mathrm{MSC}_{\text {gal-3 }} \mathbf{v}\right)$ : 5'-CAC UUU AAC CCA CGC UUC AdTdT-3' and 5'UGA AGC GUG GGU UAA AGU GdTdT-3'; negative control sequences $\left(\mathrm{MSC}_{\mathrm{NC}}\right)$ : 5'-UUC UCC GAA CGU GUC ACG UTT-3' and 5'-ACG UGA CAC GUU CGG AGA ATT-3'.

P30-MSCs were seeded in 6-well plates, and then transfected with a final concentration of $40 \mathrm{pmol} / \mu \mathrm{l} \mathrm{MSC}_{\text {gal-3 }}$ or $\mathrm{MSC}_{\mathrm{NC}}$ and incubated for $24 \mathrm{~h}$. Following the incubation and supernatant collection, total RNA or proteins were prepared and quantified as described above. Galectin-3 was quantified using the Human Galectin-3 Platinum ELISA Kit.

Moreover, the addition of recombinant galectin-3 (R\&D Systems, Minneapolis, MN, USA) with a final concentration of $50-100 \mathrm{nmol} / \mathrm{ml}$ to the $\mathrm{CM}$ of the galectin3 knockdown MSCs, and the proliferation of LoVo cells were analyzed.

\section{Western blotting for mitogen-activated protein kinase (MAPK)}

LoVo cells were incubated with CM of MSCs treated with siRNA in the presence or absence of U0126, the specific inhibitor of MEK1/MEK2. Proteins were extracted from LoVo cells using RIPA buffer, separated by $10 \%$ SDS-PAGE, and transferred to Immobilon PVDF membranes (Millipore). The membranes were blocked with PBS containing $5 \%$ non-fat dry milk and $0.1 \%$ Tween 20 overnight. After washing, the membranes were incubated with antibodies against phosphorylated extracellular signal-regulated kinase $1 / 2$ (pERK1/2) and total ERK1/2 (tERK1/2) (Cell Signaling Technology, Inc., Danvers, MA, USA), incubated with HRP-conjugated secondary antibody and visualized with SuperSignal West Pico Chemiluminescent Substrate (Millipore). 


\section{Statistical analysis}

The data are presented as the mean \pm standard deviation from $\geq 3$ experiments analyzed using SPSS 17.0 statistical software (IBM, New York, NY, USA). Analysis of significance between two groups was performed with an unpaired 2-tailed Student $t$-test; 1-way analysis of variance was used for multiple comparisons. Differences were considered significant when $P<0.05$.

\begin{abstract}
Abbreviations
ARF: ADP ribosylation factor; CM: Conditioned media; DMEM: Dulbecco's modified Eagle's medium; ELISA: Enzyme-linked immunosorbent assay; ERK: Extracellular signal-regulated kinase; FBS: Fetal bovine serum; HLA-DR: Human leukocyte antigen-DR; HRP: Horseradish peroxidase; INK4a: Cyclin-dependent kinase inhibitor 2A; MAPK: Mitogen-activated protein kinase; MSCs: Mesenchymal stem cells; P3: Passage 3; P30: Passage 30; PBS: Phosphate-buffered saline; PVDF: Polyvinyl difluoride; Q-PCR: Quantitative polymerase chain reaction; RIPA: Radioimmunoprecipitation assay; SA- $\beta$ Gal: Senescence-associated $\beta$-galactosidase; SASP: Senescence-associated secretory phenotype; SDS-PAGE: Sodium dodecyl sulfate-polyacrylamide gel electrophoresis; siRNA: Small interfering RNA; AD-MSCs: Adipose derived mesenchymal stem cells.
\end{abstract}

\section{Competing interests}

The authors declare that they have no competing interests.

\section{Authors' contributions}

Yanju $L$ designed the overall study, supervised the project, analyzed data, and wrote the manuscript. XX provided suggestions for the project, prepared the manuscript and edited the manuscript. LW and Yanqi $L$ performed siRNA assay and ELISA analysis. GL and HL performed Q-PCR and western blot analysis, $\mathrm{XW}$ and $\mathrm{YJ}$. All authors read and approved the final manuscript.

\section{Acknowledgements}

This work was supported by the State High-tech Research and Development Plans (Grants: 2011AA020109).

\section{Author details}

'Department of Hematology, Affiliated Hospital of Guiyang Medical College, No. 28, Guiyi Street, Yunyan District, Guiyang, Guizhou Province 550004, China. ${ }^{2}$ Skin Regeneration Department of General Hospital of Chinese Armed Police, No. 69 Yongding Road, Haidian District, Beijing 100039, China. ${ }^{3}$ Department of Hematology, The Second Hospital of Hebei Medical University, Hebei Key Laboratory of Hematology, Hebei Institution of Hematology, 215 West Heping Road, Shijiazhuang City, Hebei Province 050000, China. ${ }^{4}$ Yanzhou Hospital of Traditional Chinese Medicine, No. 43 Dongqiao North Road, Yanzhou District, Jining, Shandong Province 272100, China.

Received: 20 January 2015 Accepted: 23 April 2015 Published online: 06 May 2015

\section{References}

1. Collado M, Blasco MA, Serrano M. Cellular senescence in cancer and aging. Cell. 2007;130(2):223-33.

2. Campisi J, d'Adda di Fagagna F. Cellular senescence: when bad things happen to good cells. Nat Rev Mol Cell Biol. 2007:8(9):729-40.

3. Feldser DM, Greider CW. Short telomeres limit tumor progression in vivo by inducing senescence. Cancer Cell. 2007;11(5):461-9.

4. Ohtani N, Takahashi A, Mann DJ, Hara E. Cellular senescence: a double-edged sword in the fight against cancer. Exp Dermatol. 2012;21 Suppl 1:1-4.

5. Bianco P, Robey PG, Simmons PJ. Mesenchymal stem cells: revisiting history, concepts, and assays. Cell Stem Cell. 2008;2(4):313-9.

6. Akiyama K, Chen C, Wang D, Xu X, Qu C, Yamaza T, et al. Mesenchymal-stem-cell-induced immunoregulation involves FAS-ligand-/ FAS- mediated T cell apoptosis. Cell Stem Cell. 2012;10(5):544-55.

7. Cheng H, Qiu L, Ma J, Zhang H, Cheng M, Li W, et al. Replicative senescence of human bone marrow and umbilical cord derived mesenchymal stem cells and their differentiation to adipocytes and osteoblasts. Mol Biol Rep. 2011;38(8):5161-8

8. Bonab MM, Alimoghaddam K, Talebian F, Ghaffari SH, Ghavamzadeh A, Nikbin B. Aging of mesenchymal stem cell in vitro. BMC Cell Biol. 2006;7:14

9. Baxter MA, Wynn RF, Jowitt SN, Wraith JE, Fairbairn LJ, Bellantuono I. Study of telomere length reveals rapid aging of human marrow stromal cells following in vitro expansion. Stem Cells. 2004;22(5):675-82.

10. Severino V, Alessio N, Farina A, Sandomenico A, Cipollaro M, Peluso G, et al. Insulin-like growth factor binding proteins 4 and 7 released by senescent cells promote premature senescence in mesenchymal stem cells. Cell Death Dis. 2013;4:e911.

11. Efimenko A, Dzhoyashvili N, Kalinina N, Kochegura T, Akchurin R, Tkachuk V, et al. Adipose-derived mesenchymal stromal cells from aged patients with coronary artery disease keep mesenchymal stromal cell properties but exhibit characteristics of aging and have impaired angiogenic potential. Stem Cells Transl Med. 2014;3(1):32-41.

12. De Boeck $A$, Pauwels $P$, Hensen $K$, Rummens JL, Westbroek W, Hendrix A, et al. Bone marrow-derived mesenchymal stem cells promote colorectal cancer progression through paracrine neuregulin 1/HER3 signalling. Gut. 2013;62(4):550-60.

13. Song L, Tang JW, Owusu L, Sun MZ, Wu J, Zhang J. Galectin-3 in cancer. Clin Chim Acta. 2014;431:185-91.

14. Gao X, Balan V, Tai G, Raz A. Galectin-3 induces cell migration via a calcium-sensitive MAPK/ERK1/2 pathway. Oncotarget. 2014;5(8):2077-84.

15. Datta A, Loo SY, Huang B, Wong L, Tan SS, Tan TZ, et al. SPHK1 regulates proliferation and survival responses in triple-negative breast cancer. Oncotarget. 2014;5(15):5920-33.

16. Santen RJ, Song RX, McPherson R, Kumar R, Adam L, Jeng MH, et al. The role of mitogen-activated protein (MAP) kinase in breast cancer. J Steroid Biochem Mol Biol. 2002;80(2):239-56.

17. Kuilman T, Peeper DS. Senescence-messaging secretome: SMS-ing cellular stress. Nat Rev Cancer. 2009;9(2):81-94.

18. Zhang C, Zhai W, Xie Y, Chen Q, Zhu W, Sun X. Mesenchymal stem cells derived from breast cancer tissue promote the proliferation and migration of the MCF-7 cell line. Oncol Lett. 2013;6(6):1577-82.

19. Karnoub AE, Dash AB, Vo AP, Sullivan A, Brooks MW, Bell GW, et al. Mesenchymal stem cells within tumour stroma promote breast cancer metastasis. Nature. 2007:449(7162):557-63.

20. Walter M, Liang S, Ghosh S, Hornsby PJ, Li R. Interleukin 6 secreted from adipose stromal cells promotes migration and invasion of breast cancer cells. Oncogene. 2009;28(30):2745-55.

21. Zhu W, Huang L, Li Y, Qian H, Shan X, Yan Y, et al. Mesenchymal stem cell-secreted soluble signaling molecules potentiate tumor growth. Cell Cycle. 2011;10(18):3198-207.

22. Di GH, Liu Y, Lu Y, Liu J, Wu C, Duan HF. IL-6 secreted from senescent mesenchymal stem cells promotes proliferation and migration of breast cancer cells. PLoS One. 2014;9(11):e113572.

23. Radosavljevic G, Volarevic V, Jovanovic I, Milovanovic M, Pejnovic N, Arsenijevic N, et al. The roles of Galectin-3 in autoimmunity and tumor progression. Immunol Res. 2012;52(1-2):100-10

24. Wan SY, Zhang TF, Ding Y. Galectin-3 enhances proliferation and angiogenesis of endothelial cells differentiated from bone marrow mesenchymal stem cells. Transplant Proc. 2011;43(10):3933-8.

25. Liu GY, Xu Y, Li Y, Wang LH, Liu YJ, Zhu D. Secreted galectin-3 as a possible biomarker for the immunomodulatory potential of human umbilical cord mesenchymal stromal cells. Cytotherapy. 2013;15(10):1208-17.

26. Song $\mathrm{S}$, Ji B, Ramachandran V, Wang H, Hafley M, Logsdon C, et al. Overexpressed galectin-3 in pancreatic cancer induces cell proliferation and invasion by binding Ras and activating Ras signaling. PLoS One. 2012;7(8):e42699.

27. Guha P, Bandyopadhyaya G, Polumuri SK, Chumsri S, Gade P, Kalvakolanu DV, et al. Nicotine promotes apoptosis resistance of breast cancer cells and enrichment of side population cells with cancer stem cell-like properties via a signaling cascade involving galectin-3, alpha9 nicotinic acetylcholine receptor and STAT3. Breast Cancer Res Treat. 2014;145(1):5-22.

28. Baptiste TA, James A, Saria M, Ochieng J. Mechano-transduction mediated secretion and uptake of galectin-3 in breast carcinoma cells: implications in the extracellular functions of the lectin. Exp Cell Res. 2007;313(4):652-64.

29. Kim EK, Choi EJ. Pathological roles of MAPK signaling pathways in human diseases. Biochim Biophys Acta. 2010;1802(4):396-405. 
30. Hsu HH, Kuo WW, Ju DT, Yeh YL, Tu CC, Tsai YL, et al. Estradiol agonists inhibit human LoVo colorectal-cancer cell proliferation and migration through p53. World J Gastroenterol. 2014;20(44):16665-73.

31. Gieseke F, Bohringer J, Bussolari R, Dominici M, Handgretinger R, Muller I. Human multipotent mesenchymal stromal cells use galectin-1 to inhibit immune effector cells. Blood. 2010;116(19):3770-9.

Submit your next manuscript to BioMed Central and take full advantage of:

- Convenient online submission

- Thorough peer review

- No space constraints or color figure charges

- Immediate publication on acceptance

- Inclusion in PubMed, CAS, Scopus and Google Scholar

- Research which is freely available for redistribution 